\title{
Exploring Risk Factors that Contribute to the Onset of Ritodrine- Associated Serious Adverse Drug Reactions
}

Chiyo Mori ${ }^{1 *}$ Reiko Yano², Takamasa Sakai ${ }^{1}$, Jinsaku Sakakibara ${ }^{1}$, Kouichi Tanabe ${ }^{3}$, Nobuyuki Goto ${ }^{3}$ and Fumiko Ohtsu ${ }^{1}$

${ }^{1}$ Pharmaceutical Information Center, Meijo University, Tenpaku-ku, Nagoya, 468-0077, Japan

${ }^{2}$ College of Pharmacy, Kinjo Gakuin University, Moriyama-ku, Nagoya, 463-8521, Japan

${ }^{3}$ Drug Informatics, Meijo University, Tenpaku-ku, Nagoya, 468-0077, Japan

\begin{abstract}
Background: Ritodrine is a drug used for threatened premature labor. The severe adverse drug reactions associated with ritodrine are known to be pneumonedema, leukopenia, and rhabdomyolysis, but there have been few investigations on the risk factors. We performed a case-control study and selected case reports as a case group and healthy pregnant women in clinical practice as a control group.

Methods: We extracted the onsets of pneumonedema, leukopenia, and rhabdomyolysis associated with ritodrine from case reports in Japan as a case group. We selected healthy pregnant women with ritodrine administration in clinical practice as a control group. We investigated their age, medical history; Pregnancy Induced Hypertension (PIH), multiple pregnancies, concomitant drugs administered, and maximum rate of ritodrine infusion, and examined the association with those factors with the onset of adverse drug reactions by logistic regression analysis.

Results: The results of the case group showed: pneumonedema (28 cases); leukopenia (25 cases); rhabdomyolysis (21 cases). The risk factors significantly associated with pneumonedema are a medical history of the cardiovascular system, $\mathrm{PIH}$, multiple pregnancy, and concomitant treatment with steroids, which all match with the precautions in ritodrine's package insert. The factors associated with leukopenia are its administration longer than 7 days and the concomitant treatment with $\mathrm{Mg}$. The factors associated with rhabdomyolysis are multiple pregnancies and a concomitant treatment with $\mathrm{Mg}$.

Conclusion: Risk factors for the onset of pneumonedema match the descriptions in the ritodrine package insert, and can be explained by pharmacological actions. Thus, this study could elucidate the risk factors for rare adverse drug reactions limited to pregnant women. The onsets of leukopenia and rhabdomyolysis were caused by physiological changes by pregnancy and its progression of disease state and ritodrine's pharmacological action, and were suggested the possibility of risk factors.
\end{abstract}

Keywords: Ritodrine; Risk factors; Rare adverse drug reactions; Pregnant women; Case-control study; Pneumonedema; Leukopenia; Rhabdomyolysis

Abbreviations: PIH: Pregnancy Induced Hypertension; Risk Management Plan (RMP)

\section{Introduction}

It is difficult to conduct a prospective study for safety research on drug use by pregnant women due to ethical concerns. Additionally, many clinical trials exclude pregnant women and nursing women; thus, information on drug use by pregnant women is limited and difficult to obtain. Despite that, we have to contribute to appropriate drug treatment based on limited information since many patients take drugs during their pregnancy.

We conducted a case series study based on case reports in Japan and reported the characteristics of the onset of adverse drug reactions that occurred in pregnant women [1]. As a result, the most reported causative drug which leads the onset of adverse drug reaction occurred in pregnant women was ritodrine hydrochloride (ritodrine), and the most reported adverse drug reactions were pneumonedema, leukopenia, and rhabdomyolysis. Those adverse drug reactions were severe and affected fetuses in many cases. Ritodrine exhibits activity of uterine relaxation resulting from the stimulating effect of $\beta_{2}$-receptors that are highly selective for the uterus $[2,3]$, and has been frequently used for threatened premature labor in Japan.

However, the European Medicines Agency urged limited usage of the parenteral products applicable to obstetrics, such as 6-component short-acting $\beta_{2}$ stimulators, including ritodrine, to 48 $\mathrm{h}$ in maximum (between the $22^{\text {nd }}$ and $37^{\text {th }}$ weeks of pregnancy). The results of foreign comparative studies $[4,5]$ indicate that short-acting beta stimulators including ritodrine prevent delivery within $48 \mathrm{~h}$ after their administration, but were not effective for the improvement of premature birth and infant prognosis after the perinatal period. On the other hand, one multicenter study in japan showed its effectiveness in extending delivery [6], and it has been widely used since there are a limited number of alternatives to ritodrine for threatened premature birth.

Pneumonedema is a severe adverse drug reaction that has been deemed an "Important identified risk" as classified by the Risk Management Plan (RMP). The risk factors for its onset have been examined based on the results accumulated from spontaneous reports thus far. Due to this, is currently mandatory in Japan to include

*Corresponding author: Chiyo Mori, Pharmaceutical Information Center, Faculty of Pharmacy, Meijo University, 150 Yagotoyama, Tenpaku-ku, Nagoya, 468-0077, Japan, Tel: +81 052833 1063; Fax: +81 052832 8904; E-mail: 134331509@ccalumni.meijo-u.ac.jp

Received: October 29, 2016; Accepted November 30, 2016; Published December 07, 2016

Citation: Mori C, Yano R, Sakai T, Sakakibara J, Tanabe K, et al. (2016) Exploring Risk Factors that Contribute to the Onset of Ritodrine-Associated Serious Adverse Drug Reactions. J Pharmacovigil 4: 224. doi:10.4172/2329-6887.1000224

Copyright: (c) 2016 Mori C, et al. This is an open-access article distributed under the terms of the Creative Commons Attribution License, which permits unrestricted use, distribution, and reproduction in any medium, provided the original author and source are credited. 
precautions for its use in its drug package insert. Leukopenia and rhabdomyolysis are also potential risks, but their frequency of onset is unknown, and has not been elucidated much. In a situation such as this, an epidemiological study such as a cohort study or case-control study is supposed to be adopted as a method of monitoring "important identified risks" and "potential risks"; however, it is difficult to design a prospective cohort study in order to do risk management because the patients are pregnant women and the onset frequency of adverse drug reactions is rare. It is also difficult to collect a large enough number of cases into groups at a single facility to perform a case-control study.

We have developed a database entitled Case Reports of Adverse Drug Reaction Poisoning Information System (CARPIS) that has been accumulating case reports of adverse drug reactions and toxication in Japan since 1987. In our study, we regarded CARPIS as a cohort of adverse drug reaction cases and performed a case-control study in order to elucidate risk factors for the target adverse drug reactions and subjective symptoms $[7,8]$. We selected rare and severe adverse drug reactions for a case group and other case reports that excluded adverse drug reactions for a control group. In this type of study about pregnant women it is necessary that only pregnant women take part in the control group. Thus, we externally performed a case-control study by collecting data on healthy pregnant woman based on actual of obstetrics and gynecology as an external control group.

The purpose of this study is: 1) To evaluate the validity of a casecontrol study using the data of healthy pregnant women from actual clinical trials as an external control in order to elucidate the risk factors for rare adverse drug reactions in a vulnerable group such as pregnant women who cannot become a study subject of clinical research; and 2) To reveal the status of using ritodrine and patient (pregnant women) backgrounds which lead to severe adverse drug reactions by performing a case- control study.

\section{Methods}

\section{Control group}

We extracted groups with the onset of pneumonedema, leukopenia, and rhabdomyolysis from CARPIS and formed a 'pneumonedema case group', 'leukopenia case group', and 'rhabdomyolysis case group'. CARPIS is a database that has been exhaustively cumulated case reports of adverse drug reactions and toxication in Japan. We extracted case reports from 1987 to October 2014.

We selected healthy pregnant women who did not develop an adverse drug reaction after ritodrine administration as a control group. The subject facility is Medical Cooperation Kishokai Angel Bell Hospital (Angel Bell Hospital), and the criteria for patient inclusion were: 1) Patients who were hospitalized sometime between April 2013 and March 2015; 2) Those who were administered ritodrine during their hospitalization; 3) Those who did not develop pneumonedema, leukopenia, and rhabdomyolysis after ritodrine administration. All study patients were defined as the external control population. Three cases were extracted from the external control population per a case from each case group of pneumonedema, leukopenia, and rhabdomyolysis, and those cases were selected as a control group.

Angel Bell Hospital is a primary medical institution and does not accept high-risk patients. High-risk patients with following conditions are transferred to a higher order medical institution: threatened premature labor (those with a previous history of premature labor before the $34^{\text {th }}$ week of pregnancy, premature rupture of membranes, and a marked increase of inflammatory reaction), intrauterine growth restriction, Pregnancy Induced Hypertension (PIH) before the $36^{\text {th }}$ week of pregnancy, those needing a Cesarean section, partial placenta previa, multiple pregnancies, pregnancy complications (such as uterine fibroids), placental abruption, or a baby weighing less than 2,100 g. Therefore, the target patients are mainly low-risk healthy pregnant women and appropriate as an external control group.

\section{Subjects}

We investigated the following factors for the 3 case groups and each control group. Patient background of initial administration includes the age of the pregnant women (older/younger than 35 years old), the pregnancy week (before/after the $22^{\text {nd }}$ week), previous medical history (such as cardiovascular system and infertility), $\mathrm{PIH}$, and the existence/non-existence of multiple pregnancies. We investigated the state of ritodrine use with the following factors: The duration of ritodrine administration (more/less than 7days), the existence/nonexistence of concomitant drugs (magnesium formulation: $\mathrm{Mg}$; adrenal corticosteroid: steroid), and maximum infusion rate of ritodrine (more/less than $200 \mu \mathrm{g}$ ). $\mathrm{Mg}$ has indication of threatened premature labor as well as ritodrine, and is used when the effects of ritodrine are not strong enough or ritodrine cannot be used [9]. We investigated ritodrine which needs to be carefully administered when it is used in combination with $\mathrm{Mg}$ since it has been reported that $\mathrm{Mg}$ enhances the adverse drug reactions of ritodrine [10]. Steroids are used to enhance lung maturation of premature infants because of maternal administration, and pneumonedema has been reported when they are used in combination with ritodrine. Thus, careful administration is mandatory when they are used in combination with ritodrine [11].

\section{Statistical analysis}

We performed univariate logistic regression analysis on the association between the investigation factors of 3 case groups and the control group and the onset of adverse drug reaction and calculated the odds ratio (or) and a 95\% Confidence Interval (95\% CI). The significance level is $5 \%$, and we used SPSS Statistics 22 (IBM) for the statistical analysis. We applied a dummy case to the analysis when there was no relevant case in investigation item.

We performed multivariate logistic regression analysis using the substitution method on the $\mathrm{p}$-value of the items $<0.2$ in the univariate logistic regression analysis in order to adjust for confounding factors. We performed correlation analysis on each factor in order to avoid multi-collinearity for performing multivariate logistic regression analysis, and we selected one factor from the correlated factors $(r>0.4)$

\section{Ethical considerations}

This study has been approved by the Angel Bell Hospital Institutional Review Board (IRB_A_2016_003).

\section{Evaluation of the validity of a case-control study that uses a control group that includes healthy pregnant women from clinical practice as an external control}

We set pneumonedema as an index in order to evaluate the validity of a case-control study by using a control group which includes healthy pregnant women in clinical practice as an external control group. Pneumonedema associated with the use of ritodrine can be deemed an identified risk as classified by the RMP. Accordingly, precautions about its use in general and in regard to specific populations are written on the package insert based on cumulative information about adverse drug events. We compared patient backgrounds analyzed in 
this study or the results of the studies about the use of ritodrine to the description of ritodrine in its package insert. We confirmed that there is no major change in the dosage and administration of ritodrine from the initial year of our control group to the present in order to ensure the comparability of a case group and a control group. The indications and dosage of ritodrine are as follows:

The indication for ritodrine (effect-efficacy) [2]: Threatened miscarriage or premature labor that requires emergency treatment. Dosage amount of ritodrine (dosage and administration) [2]: Dilute 1 ample $(5 \mathrm{~mL})$ of ritodrine with $5 \%$ of glucose injection or with $10 \%$ of maltose $(500 \mathrm{~mL})$; Administer ritodrine hydrochloride at $50 \mu \mathrm{g}$ per minute intravenously; Adjust increasing or decreasing the dosage accordingly by observing tocolytic activity and maternal heart rate. Gradually decrease the dosage amount while observing indication after uterine contractions are controlled; Discontinue administration while keeping the dosage amount less than $50 \mu \mathrm{g}$ per a minute and when recurrent contractions are not observed. The efficacious dose of ritodrine is $0-150 \mu \mathrm{g}$ per minute. Meanwhile, the dosage amount should not exceed $200 \mu \mathrm{g}$ per minute.

\section{Results}

The results from each group are as follows: pneumonedema: 28 cases for the case group and 84 cases for the control group; leukopenia: 25 cases for the case group and 75 cases for the control group; rhabdomyolysis: 21 cases for the case group and 63 cases for the control group.

\section{A consideration of the validity of a case control study using control group of healthy pregnant women based on actual trials as an external control and the association between pneumonedema which is an identified risk and patient backgrounds/state of ritodrine usage}

The risk factors for pneumonedema written in the precautions section of the ritodrine package insert are heart disease, a complication of PIH, multiple pregnancies, and adrenal corticosteroid if administered in combination. The case reports from the case group from 1989 to 2014 showed no change in the amount of ritodrine used or the indication of threatened premature labor.

Figure 1 shows the results of univariate logistic regression analysis on the onset of pneumonedema and investigation items. Significant factors of patient backgrounds that could be associated with adverse drug reaction were a previous medical history of cardiovascular system, a history of infertility, PIH, and multiple pregnancies. The significant factors of state of ritodrine usage were a concomitant treatment with $\mathrm{Mg}$ or steroids. Figure 2 shows the results of multivariate logistic regression analysis on 6 factors where the p-value is less than 0.2 by univariate analysis. The results did not reveal any correlation between factors. Significant associations found in patient backgrounds were a previous history of cardiovascular system (OR: 22.62, 95\% CI: $1.2-$ 413.7), PIH (OR: 28.24, 95\% CI: 3.9-205.5), and multiple pregnancy (OR: 170.59, 95\% CI: 13.0-2238.5). A significant association found in state of ritodrine usage was when it was used in combination with steroids (OR: 29.79, 95\% CI: 1.9-467.0). Those results are exactly the same in content as the risk factors written in package insert of ritodrine.

The association between potential risks such as leukopenia/ rhabdomyolysis, and patient backgrounds/state of ritodrine usage

Figure 3 shows the results of a univariate logistic regression analysis of leukopenia and investigation factors. Significant associations found in patient background were a history of infertility and multiple pregnancies. Those found in state of ritodrine usage were cases in which it was administered longer than a week, where there was a concomitant treatment with $\mathrm{Mg}$, and cases where the maximum infusion rate exceeded $200 \mu \mathrm{g}$.

Figure 4 shows the results of multivariate logistic regression analysis on 6 factors which p- value is less than 0.2 by univariate analysis. The results did not reveal any correlation between factors. Significant associations found in patient background were cases where ritodrine was administered for longer than a week (OR: 15.29, 95\% CI: 1.3-183.9) and a concomitant treatment with Mg (OR: 28.64, 95\% CI: 2.4-341.5).

Figure 5 shows the results of univariate logistic regression analysis on rhabdomyolysis and investigation factors. Significant association found in patient background was multiple pregnancies, and when ritodrine was used concomitantly with $\mathrm{Mg}$.

Figure 6 shows the results of multivariate logistic regression analysis on 2 factors where the p-value is less than 0.2 by univariate analysis. The results did not reveal any correlation between factors. A significant association found in patient background was multiple pregnancy (OR: 17.63, 95\% CI: 1.7-179.4), and that found in state of ritodrine usage was a concomitant treatment with Mg (OR: 23.43, 95\% CI: 2.5-224.2).

\section{Discussion}

We performed a case-control study with a control group of healthy pregnant women who could not ethically participate in clinical research in order to elucidate the risk factors of rare adverse drug reaction.

The factors that lead to the onset of pneumonedema associated with ritodrine and showed significant associations were a medical history of cardiovascular system, $\mathrm{PIH}$, multiple pregnancies, and a concomitant treatment with steroids.

The strain on the heart of mothers during pregnancy is increased more than usual as circulatory blood volume, cardiac output, and blood flow are increased [12]. The medical history of the cardiovascular system includes some cases with cardiovascular-related diseases in the past that can affect the strain on the heart. It has been reported that ritodrine affects myocardial performance [13], and it increases the strain on the heart. It is considered that the beta stimulant activity of ritodrine causes renin angiotensin system activation, which increases in the secretion of circulating antidiuretic hormones inside the body of pregnant women. As a result, it is likely to cause water retention and lower diuretic action $[14,15]$.

$\mathrm{PIH}$ is the symptom of which the strain on systemic vascular is increased by high-blood pressure, and increased vascular permeability by increase of inflammatory cytokine is likely to lead to water emigration outside of the blood vessels [16]. The beta stimulant activity of ritodrine leads to renin angiotensin system activation, enhances $\mathrm{Na}$ and water reabsorption, and promotes accumulation of extracellular water, which could cause the onset of pneumonedema.

Multiple pregnancies expand the uterine wall to accommodate the increasing number of fetuses, and are likely to develop into threatened premature labor. Twin fetuses increase plasma volume [17], and Lamont noted that twin fetuses are theoretically sensitive to the influence of blood transfusion by tocolysis [15]. The increase in circulatory blood volume associated with the increasing number of fetuses increases the strain on renal functioning; additionally, the action of ritodrine is a factor that worsens the physiological changes that come with multiple pregnancies. Those factors could be considered to affect the onset of pneumonedema. 
Citation: Mori C, Yano R, Sakai T, Sakakibara J, Tanabe K, et al. (2016) Exploring Risk Factors that Contribute to the Onset of Ritodrine-Associated Serious Adverse Drug Reactions. J Pharmacovigil 4: 224. doi:10.4172/2329-6887.1000224

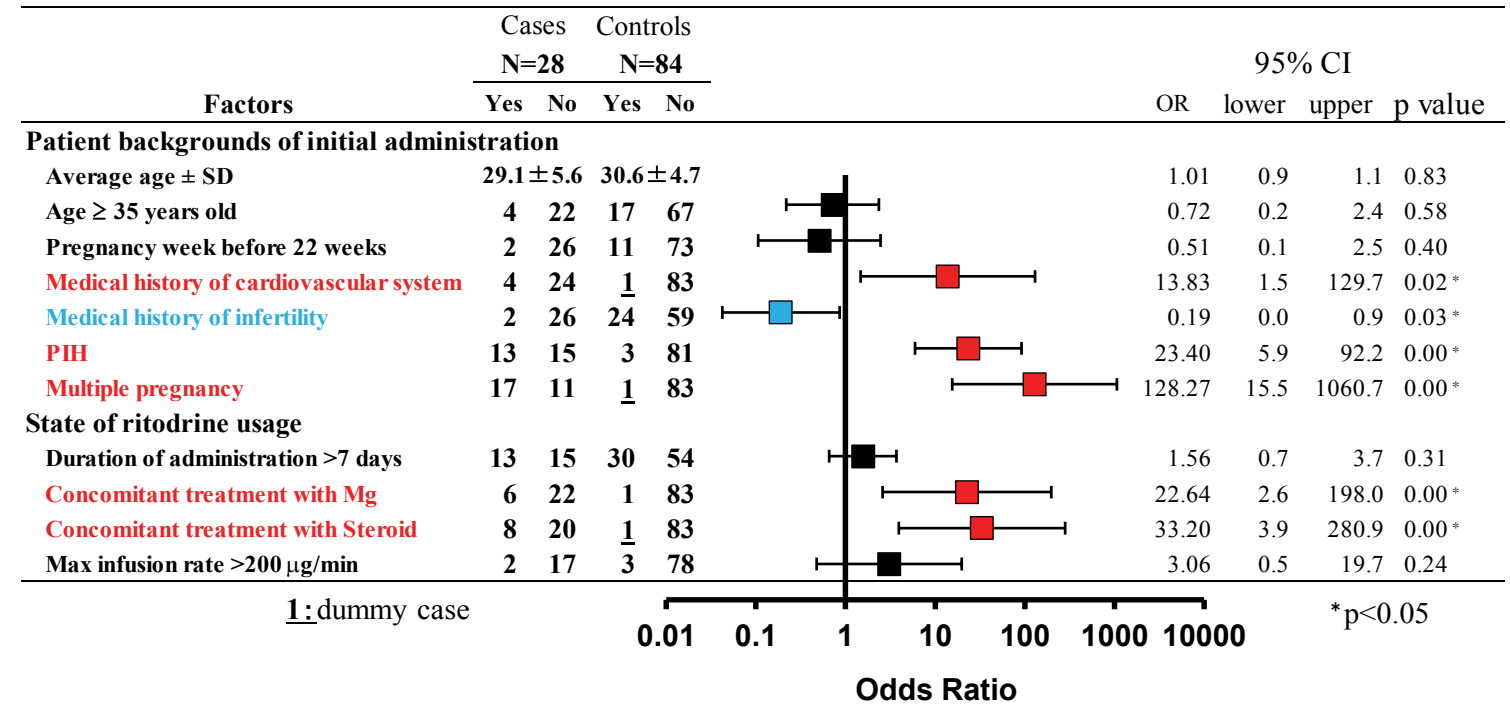

Figure 1: The association between pneumonedema and patient backgrounds/Ritodrine usage by univariate logistic regression analysis.

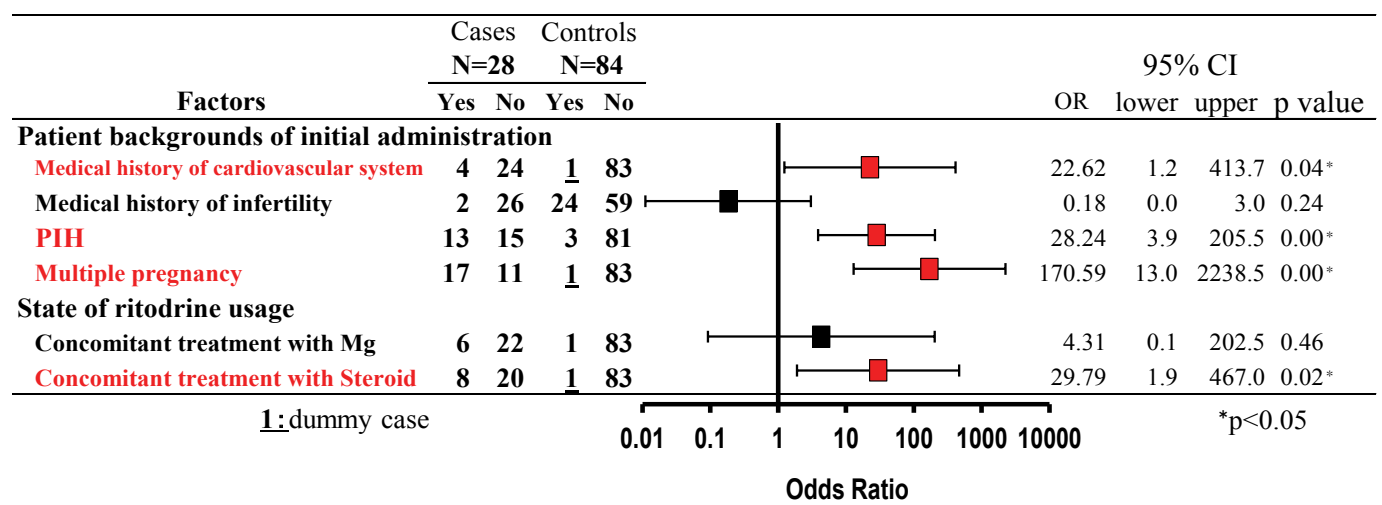

Figure 2: The association between pneumonedema and patient backgrounds/Ritodrine usage by multivariate logistic regression analysis.

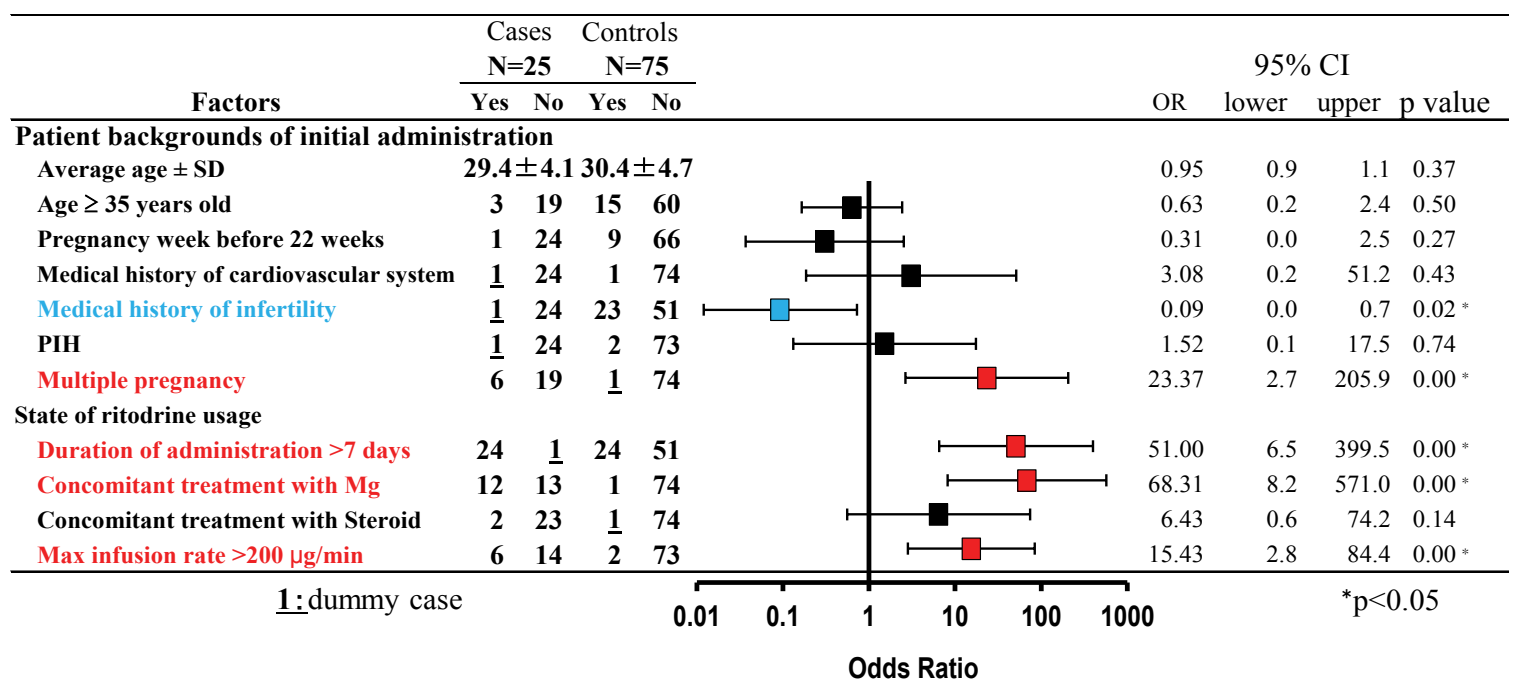

Figure 3: The association between leukopenia and patient backgrounds/Ritodrine usage by univariate logistic regression analysis. 
Citation: Mori C, Yano R, Sakai T, Sakakibara J, Tanabe K, et al. (2016) Exploring Risk Factors that Contribute to the Onset of Ritodrine-Associated Serious Adverse Drug Reactions. J Pharmacovigil 4: 224. doi:10.4172/2329-6887.1000224

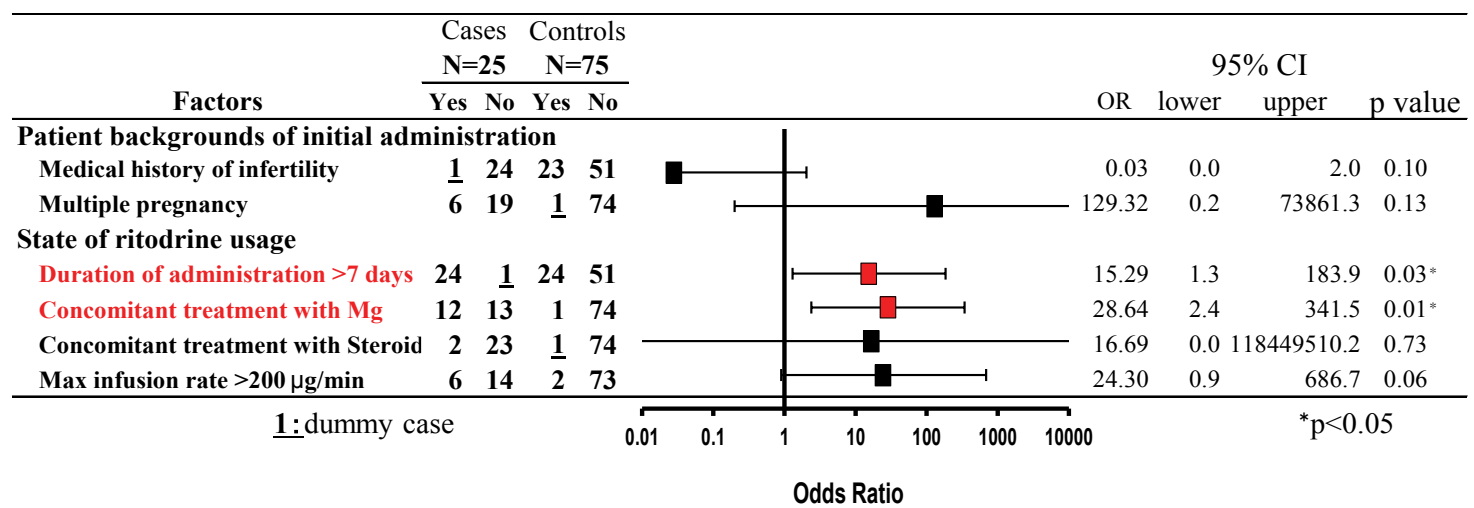

Figure 4: The association between leukopenia and patient backgrounds/Ritodrine usage by multivariate logistic regression analysis.

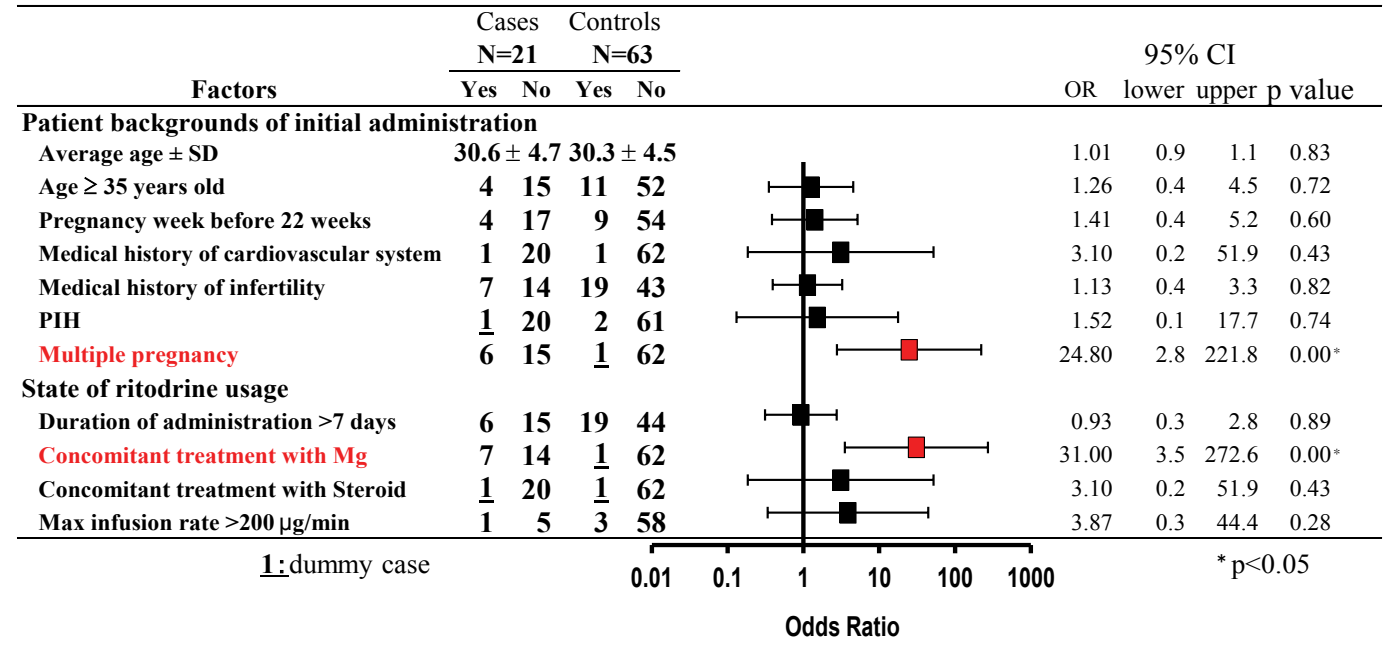

Figure 5: The association between rhabdomyolysis and patient backgrounds/Ritodrine usage by univariate logistic regression analysis.

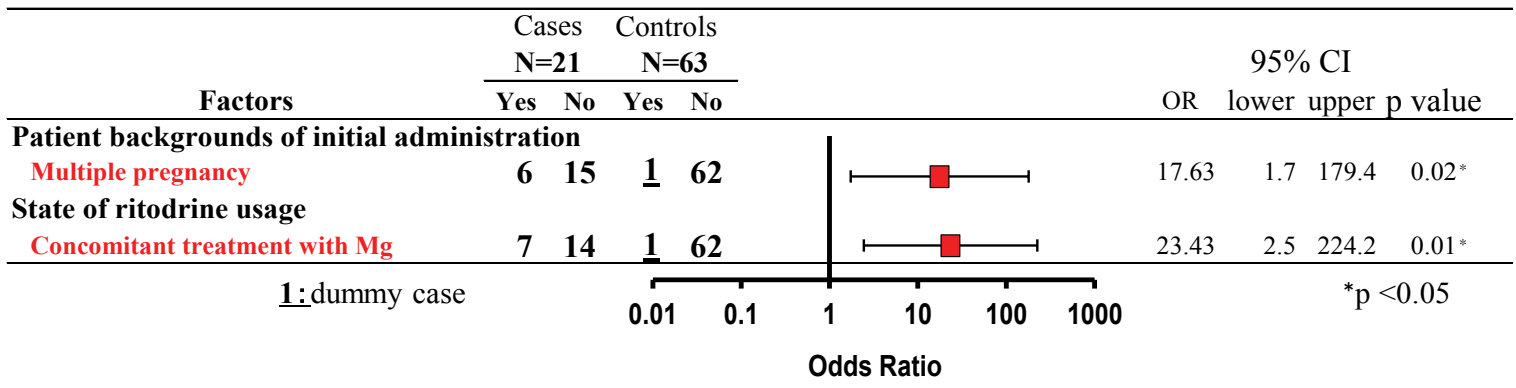

Figure 6: The association between rhabdomyolysis and patient backgrounds/Ritodrine usage by multivariate logistic regression analysis.

The concomitant treatment with steroids could enhance water retention inside of the body, which could lead to the onset of pneumonedema [11]. The mineralocorticoid activity of steroids increases the water volume in the body because the activity promotes $\mathrm{Na}$ and water reabsorption. The beta stimulant activity of ritodrine is likely to cause water retention and lower diuretic action because the renin angiotensin system is activated [14,15], and a concomitant treatment with steroids is likely to cause the development of pneumonedema.

The factors found in this study: medical history of cardiovascular issues, $\mathrm{PIH}$, multiple pregnancies, and a concomitant treatment with steroids match the descriptions of the items listed in the Precautions section of the package insert which are cardiac diseases, $\mathrm{PIH}$, multiple pregnancies, and a concomitant treatment with adrenal corticosteroids. These factors are risk factors for the onset of pneumonedema, an identified risk of ritodrine as a pharmacological function. This study that extracted these factors appropriately could have high validity as a method of identifying risk factors for adverse drug reactions whose expression frequency is low and in pregnant women where a clinical study in a real world setting is unlikely to be designed. 
Citation: Mori C, Yano R, Sakai T, Sakakibara J, Tanabe K, et al. (2016) Exploring Risk Factors that Contribute to the Onset of Ritodrine-Associated Serious Adverse Drug Reactions. J Pharmacovigil 4: 224. doi:10.4172/2329-6887.1000224

Page 6 of 7

On the other hand, we could obtain information about potential risks, such as leukopenia and rhabdomyolysis, which had previously been insufficient. The factors that showed significant association with the onset of leukopenia associated with ritodrine administration were when it was administered longer than 7 days and a concomitant treatment with $\mathrm{Mg}$. The number of leucocytes would be increased according to the increasing number of polymorphonuclear neutrophil leukocytes and myelocytes at the time of pregnancy [12]. However, the development of chorioamnionitis, which is considered one of the factors that causes premature labor, consumes a high volume of leukocytes in order to cure the inflammation it causes. It is suggested that ritodrine could cause leukopenia because of dosage dependence in the periphery, cytotoxicity which inhibits the growth of colony-forming unitgranulocyte macrophage (CFU-GM), and immunological mechanisms which cause the destruction of granulocytes by antineutrophil antibody production [18]. Bone-marrow cells form colonies [19] and become differentiated into granulocytes and macrophages after 7-14 days, but if ritodrine administration is extended for a longer period than 7-14 days, leukocyte production drastically decreases. Toxic activity of ritodrine continues against granulocytes and macrophages during its administration and at the same time, develops in parallel with their destruction. Therefore, it is inferred that leukopenia could develop drastically when ritodrine administration is extended longer; leukopoiesis would decrease; the pace of consuming and destroying would increase.

There are no reports that $\mathrm{Mg}$ affects leukocyte production. A concomitant treatment with ritodrine and $\mathrm{Mg}$ caused rapid symptoms of neutropenia, but there is a report that the neutrophil count recovered when ritodrine was discontinued and $\mathrm{Mg}$ was continued as a singleagent treatment [20]. A concomitant treatment with $\mathrm{Mg}$ is particularly used in the case that the amount of ritodrine administration was increased because of serious threatened abortion. Therefore, there is a significant association with a risk of severe adverse events and the concomitant treatment with $\mathrm{Mg}$ and an extended period of ritodrine administration.

The factors of the onset of rhabdomyolysis significantly associated with ritodrine administration were multiple pregnancies and a concomitant treatment with $\mathrm{Mg}$. Ritodrine promotes $\mathrm{Ca}^{2+}$ in the cell to take inside of the body after attaching oneself to the receivers and to relax uterine smooth muscle by inactivating myosin light chain kinase [1]. Drastic changes in potassium concentration inside the cell cause instability in muscle-cell membrane and increase the probability of muscle cell damage [21].

Multiple pregnancies are one risk factor for threatened premature labor, but there have been no reports that show a clear association with multiple pregnancies and the onset of rhabdomyolysis. This is because the dosage amount of ritodrine is increased as threatened premature labor worsens.

$\mathrm{Mg}$ increases uterotonic activity associated with ritodrine [9], promotes changes in $\mathrm{Ca}^{2+}$ concentration in the cells and destabilizes the cell membrane because of electrolyte abnormality; thus, it is possible that $\mathrm{Mg}$ could cause muscle cell damage [21]. A concomitant treatment with $\mathrm{Mg}$ enhances ritodrine's effect on muscle cells; thus, rhabdomyolysis might be likely to occur.

Our study suggested that a variety of the pharmacological actions of ritodrine and physiological changes in pregnant women were particular risk factors for the onset of severe adverse drug reactions associated with ritodrine.

\section{Limitations of this Study}

We considered that this study was supposed to investigate the dosage amount of ritodrine, its duration of use, and drug absorption rate; however, we could not investigate sufficient data because most case reports did not include descriptions of the dosage amount and the infusion rate. The odds ratio of the identified factors in this study showed a wide range with a $95 \%$ CI. We need to investigate a larger number of cases for further study.

The patient background in the factors we investigated includes pregnant women with high risk factors, and those patients need to be taken care of by higher order medical institutions. The control group in this study was extracted from a primary medical institution, and the patients with high risk factors are transferred to higher order medical institutions in accordance with the criteria of patient transportation before their symptoms worsen. Therefore, the frequency of the onset of severe adverse drug reactions is likely to be less than general occurrence frequency and it is thus possible to over evaluate the risk. On the other hand, it is extremely difficult to detect the onset of severe adverse drug reactions associated with ritodrine early and act promptly and appropriately because they are rare even in clinical practice. The recognition of risk factors found in this study which affect the onset of adverse drugs reactions could lead early detection in clinical practice although it might be possible to over- evaluate the risk factors due to their rarity at general medical institutions.

\section{References}

1. Mori C, Sakai T, Yano R, Tanabe K, Goto N, et al. (2017) Characteristics of adverse events in pregnant women: A case series study based on case reports in Japan. Jpn J Drug Inform 18.

2. Kissei Pharmaceutical Co., LTD. (2015) Utemerine ${ }^{\circledR}$ Injection 50 mg InterviewForm.

3. European Medicines Agency (EMA) (2013) Restrictions on use of short-acting beta-agonists in obstetric indications-CMDh endorses PRAC recommendations. London, UK.

4. Anotayanonth S, Subhedar NV, Garner P, Neilson JP, Harigopal S (2004) Betamimetics for inhibiting preterm labour. Cochrane Database Syst Rev.

5. The Canadian Preterm Labor Investigators Group (1992) Treatment of preterm labor with the beta-adrenergic agonist ritodrine. N Eng J Med 327: 308-312.

6. Sato K, Terao T, Fujimoto S, Murata Y, Ikenoue K (2006) Current state of preterm labor treatment in Japan by the multicenter study. Obstetrical and gynecological practice 55: 993-1001.

7. Hayashi K, Ohtsu F, Yano R, Sakakibara J, Goto N (2011) Risk Factors and Subjective Symptoms of Drug-induced Leucopenia. Yakugaku Zasshi: J Pharm Soc Japan 131: 139-152.

8. Hayashi K, Ohtsu F, Yano R, Sakakibara J, Goto N (2012) Study on Characteristics and Risk Factors of Adverse Drug Reactions in the Elderly Using the Database of Adverse Drug Reactions. J Pharm Health Care Sci 38: 67-77.

9. Toa Pharmaceutical Co., Ltd. (2015) Magsent ${ }^{\circledR}$ Injection $100 \mathrm{ml}$, Magsent ${ }^{\circledR}$ Injection Syringe $40 \mathrm{ml}$ Interview Form.

10. Ferguson JE, Hensleigh PA, Kredenster D (1984) Adjunctive use of magnesium sulfate with ritodrine for preterm labor tocolysis. Am J Obstet Gynecol 148: 166-171.

11. Shionogi and Co., LTD. (2015) Rinderon ${ }^{\circledR}$ Injection $2 \mathrm{mg}(0.4 \%)$, Rinderon ${ }^{\circledR}$ Injection $4 \mathrm{mg}(0.4 \%)$, Rinderon ${ }^{\circledR}$ Injection $20 \mathrm{mg}(0.4 \%)$. Interview Form.

12. Costantine MM (2014) Physiologic and pharmacokinetic changes in pregnancy Front Pharmacol 5: 65.

13. Ikeda S, Tamaoki H (1984) Pharmacological Investigation of Ritodrine Hydrochloride, a $\beta_{2}$ - Adrenoceptor Stimulant. Japan J Pharmacol 36: 477-484.

14. Armson BA, Samuels P, Miller F, Verbalis J, Main EK (1992) Evaluation of maternal fluid dynamics during tocolytic therapy with ritodrine hydrochloride and magnesium sulfate. Am J Obstet Gynecol 167: 758-765.

15. Lamont RF (2000) The Pathophysiology of pulmonary oedema with the use of beta-agonists. BJOG 107: 439-444. 
Citation: Mori C, Yano R, Sakai T, Sakakibara J, Tanabe K, et al. (2016) Exploring Risk Factors that Contribute to the Onset of Ritodrine-Associated Serious Adverse Drug Reactions. J Pharmacovigil 4: 224. doi:10.4172/2329-6887.1000224

16. Benedetti TJ, Carlson RW (1979) Studies of colloid osmotic pressure in pregnancy-induced hypertension. Am J Obstet Gynecol 135: 308-311.

17. Campbell DM, MacGillivray I (1977) Maternal physiological responses and birthweight in singleton and twin pregnancies by parity. Eur J Obstet Gyneco Reprod Biol 7: 17-24.

18. Yasuda R, Makino Y, Matsuda Y, Kawamichi Y, Matsui H (2012) Agranulocytosis associated with intravenous ritodrine hydrochloride therapy: two case reports by different mechanisms. J Obstet Gynaecol Res 38: 574-577.
19. Braunwald E (2003) Harrison's Principles of Internal Medicine ( $\left(1^{\text {st }}\right.$ edn.) Medical Sciences International, Tokyo, pp: 676-677.

20. Onoe T, Takahashi $Y$, Noda $Y(1995)$ Ritodrine-induced Neutropenia in two cases of threatened premature delivery. Acta Obst Gynecol Jpn 47: 283-286.

21. Sholl JS, Hughey MJ, Hirshmann RA (1985) Myotonic muscular dystrophy associated with ritodrine tocolysis. Am J Obstet Gynecol 151: 83-86. 